TP Periodica Polytechnica

Transportation Engineering

47(2), pp. 131-135, 2019

https://doi.org/10.3311/PPtr.11490

Creative Commons Attribution (1)

RESEARCH ARTICLE

\section{Novell Application of CFD for Rocket Engine Nozzle Optimization}

\author{
Csaba Jéger ${ }^{1}$, Árpád Veress $^{2^{*}}$
}

Received 19 January 2017; accepted 20 September 2017

\begin{abstract}
Numerical analyses, validation and geometric optimization of a converging-diverging nozzle flows has been established in the present work. The optimal nozzle contour for a given nozzle pressure ratio and length yields the largest obtainable thrust for the conditions and thus minimises the losses. Application of such methods reduces the entry cost to the market, promote innovation and accelerate the development processes. A parametric geometry, numerical mesh and simulation model is constructed first to solve the problem. The simulation model is then validated by using experimental and computational data. The optimizations are completed for conical and bell shaped nozzles also to find the suitable nozzle geometries for the given conditions. Results are in good agreement with existing nozzle flow fields. The optimization loop described and implemented here can be used in the all similar situations and can be the basis of an improved nozzle geometry optimization procedure by means of using a multiphysics system to generate the final model with reduced number sampling phases.
\end{abstract}

\section{Keywords}

rocket engine, converging-diverging nozzle flow, validation, optimization, CFD

\footnotetext{
${ }^{1}$ Department of Aeronautical and Vehicle Engineering School of Engineering Sciences, Royal Institute of Technology, SE-100 44 Stockholm, Sweden

${ }^{2}$ Department of Aeronautics, Naval Architecture and Railway Department, Faculty of Transport and Vehicle Engineering, Budapest University of Technology and Economics, H-1111 Budapest, Müegyetem rkp. 3, Hungary

*Corresponding author, e-mail: averess@vrht.bme.hu
}

\section{Introduction}

The different simulation methodologies are widely spread in many segments of the vehicle engineering as naval architecture (e.g.: Schweighofer et al. (2015)), energetic and propulsion systems (e.g.: Beneda (2012)) and aircraft structures (e.g.: Rohács at al. (2016)) for example because significant amount of cost, capacity and time can be saved by these approaches. Hence, the CFD (Computational Fluid Dynamics) based optimization process has been introduced in the present case for thrust maximisation of rocket engine nozzle flow.

Design and developments of rocket engine nozzle flow started at middle of last century. Guderley and Hantsch (1955) solved the problem of finding optimal nozzle contours with prescribed nozzle length and ambient conditions which are usually design constraints, using variational calculus. The method was complicated and not widely used until it was simplified by Rao (1958) after it became known as the Rao nozzle or Thrust Optimized Contour (TOC) nozzle. It was also suggested by Rao that the Thrust Optimized Parabolic (TOP) geometry could be approximated by a skewed parabolic contour with only minor difference in performance (1960). Since TOP solutions only yield discrete points, functional representation of the nozzle geometry can offer advantages in design. One of these representations is called Parabolic Bell Nozzle (PBN). Direct nozzle optimization using numerical tools was carried out by Cai et al. (2007) who modelled and optimized the performance of nozzles operating in vacuum thus the computational domain only consisted of the nozzle.

A new application of the CFD and optimisation method is established in the present case for process development for optimization converging-diverging nozzle flow by expecting the highest level of accuracy. This work is divided into two parts. First, a validation and verification of the nozzle model using experimental and computational data is presented. Afterwards the paper deals with the optimization of nozzle contours for having the maximum thrust by using the numerical model to be constructed.

The nozzle optimization problem can be defined as the following: given a set of external parameters and geometric constrains, find a nozzle wall contour such as the resulting thrust 
produced by the nozzle is maximal. The external parameters are: ambient pressure, temperature and chamber pressure, temperature. The geometric constraints are the nozzle length and throat diameter. Air as ideal gas has been used for the operational fluid. Validation and verification is carried out using published experimental (Torngren, 2002) and computational (Luedeke and Filimon, 2006) data on the VAC S6S test nozzle, which is an air-operated Truncated Ideal Contour (TIC) nozzle. The nozzle has a throat diameter of $67.08 \mathrm{~mm}$ and an exit diameter of $250.3 \mathrm{~mm}$ yielding an expansion ratio of 13.9. Regarding the optimization process described in Section 3, the converging and throat sections of the nozzle are kept to be constant and the optimization is carried out on the diverging part of the geometry using one and two control points.

\section{Numerical Modelling and Simulation 2.1 Flow Domain}

The throat section of the nozzle for validation is based on the VAC S6S nozzle (Torngren, 2002) using a point-tracing to obtain the geometry. Additional smoothing and curve fitting was employed so that the geometry can be generated with an arbitrary amount of control points. The points are then used to generate a coordinate file that can be used in the modelling environment. This geometry will be used also in the optimization as initial geometry.

To account for the effects of supersonic nozzle exhaust a large open area is modelled both upstream and downstream of the nozzle. The nozzle inlet section is also extended upstream of the nozzle to properly model the stagnation conditions at the inlet section. For a simpler handling of the geometry the height, upstream open length, downstream length and inlet distance from the nozzle can be controlled via parameters. Principal dimensions are set to $5 \mathrm{~m}$ length and $3 \mathrm{~m}$ height. The chamber inlet is $1 \mathrm{~m}$ upstream. The origin of the coordinate system is located on the nozzle axis in the throat section. This model is used also in the parameter sensitivity analyses.

\subsection{Numerical Mesh}

The used mesh is $2 \mathrm{D}$, unstructured and axisymmetric. The region near the axis has smaller cells to capture details of the plume pattern. The throat region is the densest part of the mesh (see Fig. 1). An inflation layer is applied to the nozzle wall. Edge lengths inside the nozzle are set to be $2 \cdot 10^{-3} \mathrm{~m}$ but there is also a denser hemispherical area originating from the axial exit point with cell edge lengths of $1.5 \cdot 10^{-3} \mathrm{~m}$. This is the region where the normal shock can appear during overexpanded operation. The boundary layer consists of 20 cells with a total thickness of $\sim 0.6$ $\mathrm{mm}$. Total cell number for the baseline mesh used in the validation process is 119685 . The modified grids used in mesh and model sensitivity studies vary from that. The mesh generated during the optimization process is similar to the baseline mesh and cell numbers vary less than $5 \%$ from the baseline.

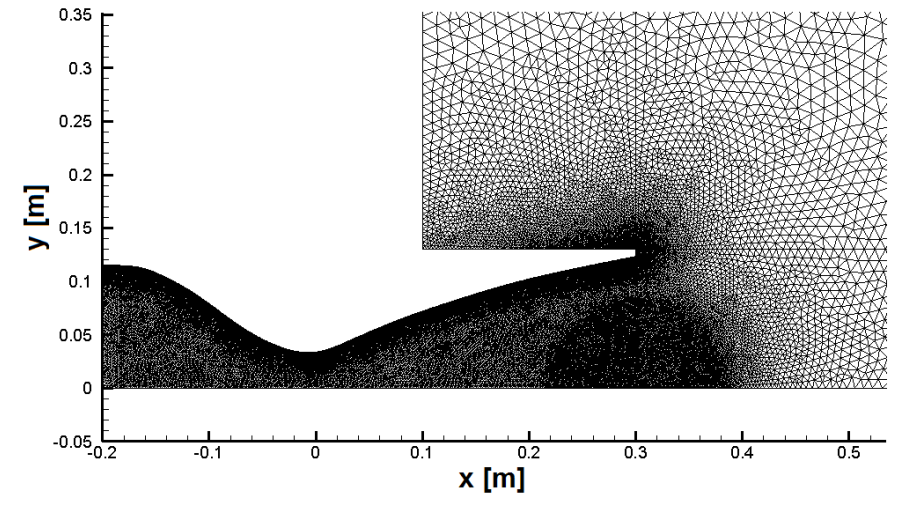

Fig. 1 Mesh close-up on the nozzle area

\subsection{Material Properties, Boundary Conditions and Solver Settings}

The working fluid is single-phase air as ideal gas in all cases. Initial Courant number was set to 1 and the flow field was initialised using hybrid initialisation. The locations and the types of the boundary conditions are shown in Fig. 2. Implicit density-based axisymmetric solver was used with full second-order upwind discretisation using the Roe FluxDifference Splitting scheme for cell wall flux calculations and Cell-Based Least Squares method for the gradients. The turbulence model used is the Shear Stress Transport model, coupled with the total energy equation. The present settings are used in the all simulations afterwards.

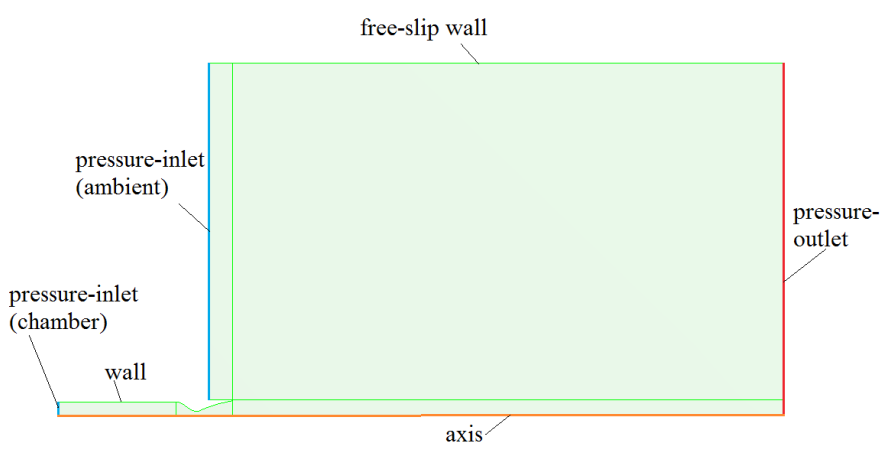

Fig. 2 Boundary conditions

\section{Validation and Verification}

The physical parameters of the boundary conditions to be considered for validation are found in Table 1. Following the converged steady state conditions, the comparison between the measured and simulated data is made using the wall pressure profiles normalized with the chamber pressure. The available experimental and numerical data is plotted along with the present results (see Fig. 3). The presently calculated normalized pressure profiles are in agreement with the validation and verification datasets, particularly in the lower Nozzle Pressure Ratio (NPR) range for the investigated case. 
Table 1 Boundary conditions for validation

\begin{tabular}{ll}
\hline Boundary name & Parameters \\
\hline pressure-inlet (chamber) & $\begin{array}{l}\mathrm{p}_{\text {total }}=2100000 \mathrm{~Pa}, \mathrm{~T}_{\text {total }}=400 \mathrm{~K} \text {, air as ideal } \\
\text { gas }\end{array}$ \\
pressure-inlet (ambient) & $\mathrm{p}_{\text {total }}=100000 \mathrm{~Pa}, \mathrm{~T}_{\text {total }}=300 \mathrm{~K}$, air as ideal gas \\
free-slip wall & adiabatic, zero shear \\
wall & adiabatic, no slip \\
pressure-outlet & $\mathrm{p}_{\text {total }}=100000 \mathrm{~Pa}$ \\
\hline
\end{tabular}

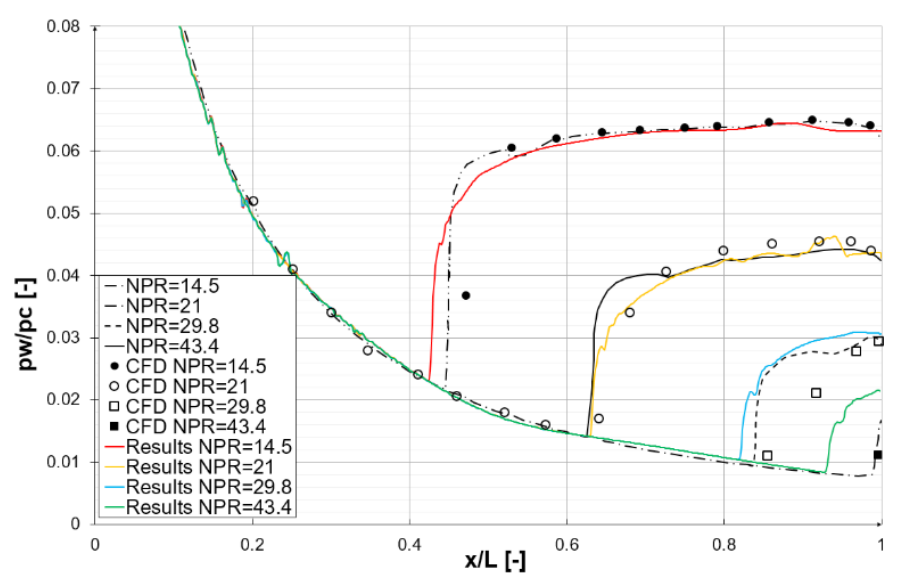

Fig. 3 Comparison of wall pressure profiles measured (black lines, (Torngren, 2002)), CFD (points, (Luedeke and Filimon, 2006)) and the results of this work (coloured lines)

To compare the wall pressure results with the experimental data, the normalised $\mathrm{L}^{2}$ norm is calculated. Results are shown in Table 2. The average value of the $\mathrm{L}^{2}$ norm is $3.6 \%$, which is below the $5 \%$, hence the results are acceptable in technical point of view.

Table 2 Normalised $\mathrm{L}^{2}$ norm of the wall pressure

\begin{tabular}{ll}
\hline NPR & $\mathrm{L}^{2}$ (experimental) [\%] \\
\hline 14.5 & 2.78 \\
21 & 1.18 \\
29.8 & 2.98 \\
43.4 & 7.50 \\
\hline
\end{tabular}

\subsection{Parameter Sensitivity Studies}

The geometry is checked to not interfere with the flow in the nozzle. Parameters were found independent for the relatively small $3 \times 5 \mathrm{~m}$ ambient flow field. The mesh was also checked for independence with mesh resolutions at 300,000 600,000 and 900,000 cells. The effect of discretisation schemes was also studied and it was found that the first-order upwind scheme predicted the flow separation prematurely.

\section{Nozzle Optimization}

The optimal geometry depends on the type of nozzle used, which translates into the number of internal control points used. In the present work a 1-parameter conical nozzle case and a 2-parameter parabolic nozzle case are concerned. In both cases the boundary conditions are the same and are summarised in Table 3. Boundary conditions are associated with the upper limit of the test samples, which is at NPR $=59.7$. Both the working fluid and ambient gas is air as ideal gas in the all simulations. The goal function in the both investigated cases is to get the maximum thrust meanwhile the shapes and the outlet cross sections of the diffusers are the parameters.

Table 3 Boundary conditions at nozzle optimization

\begin{tabular}{ll}
\hline Boundary name & Parameters \\
\hline pressure-inlet (chamber) & $\mathrm{p}_{\text {total }}=2400000 \mathrm{~Pa}, \mathrm{~T}_{\text {total }}=400 \mathrm{~K}$, air as ideal gas \\
pressure-inlet (ambient) & $\mathrm{p}_{\text {total }}=40201 \mathrm{~Pa}, \mathrm{~T}_{\text {total }}=300 \mathrm{~K}$, air as ideal gas \\
free-slip wall & adiabatic, zero shear \\
wall & adiabatic, no slip \\
pressure-outlet & $\mathrm{p}_{\text {total }}=40201 \mathrm{~Pa}$ \\
\hline
\end{tabular}

\subsection{Conical Nozzle: 1-parameter Optimization}

Only the exit diameter is design parameter in this case. Up to the throat the geometry is fixed. There is an extra point downstream of the throat section which is placed to smooth the transition between the spline of the confusor section and the linear segment of the diffusor trough a tangential condition. The diameter of the nozzle is controlled between 0.16 and 0.20 meters. The Central Composite Design (CCD) method generates 5 design points which are then used by a non-parametric regression to construct the response surface, which is one-dimensional here. Thrust is derived from the diameter (input parameter) and simulation outputs (mass flow rate, mean axial exit velocity and pressure difference). The optimum condition response is plotted in Fig. 4. The function has a well observable minimum at approximately $\mathrm{d}_{\mathrm{e}}=0.164 \mathrm{~m}$ outlet diameter. The results show that the flow at the optimum expansion condition and a weak oblique shock is observable in the plume which is in agreement with the internal flow fields produced by other conical nozzles. The flow field of the optimized nozzle is found in Fig. 5.

\subsection{Bell nozzle: 2-parameter Optimization}

The diffusor section of the geometry is represented with a second-order parabolic curve where the nozzle diameter $d_{e}$ and the " $y$ " coordinate of the point $\mathrm{P}_{1}$ halfway downstream from the throat are the design variables and the thrust condition is evaluated in a $2 \mathrm{D}$ design space. The parameter $\mathrm{P}_{1}$ varies between 0 and 1 in a way that at the minimum, it becomes a conical geometry and at maximum the diameter still has the maximum at the nozzle exit. The diameter of the nozzle is controlled between 0.16 and 0.20 meters as before. The CCD method generates 9 


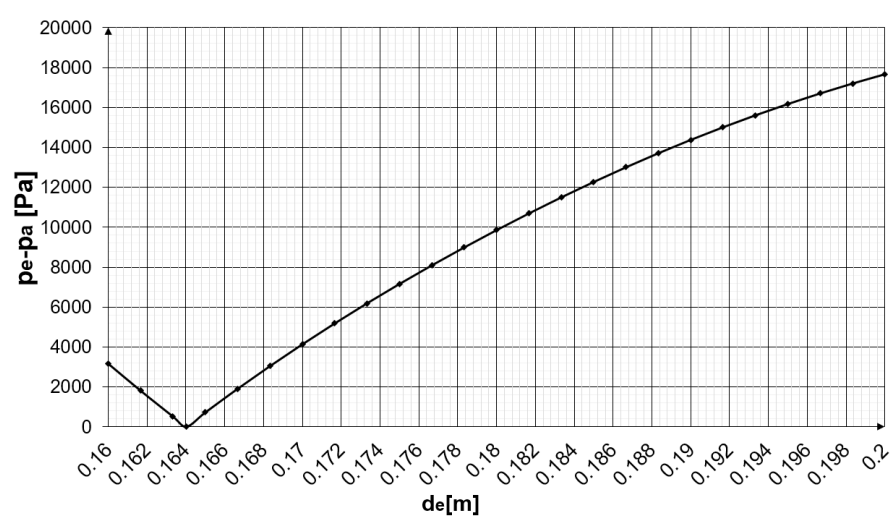

Fig. 4 Pressure difference response, conical nozzle

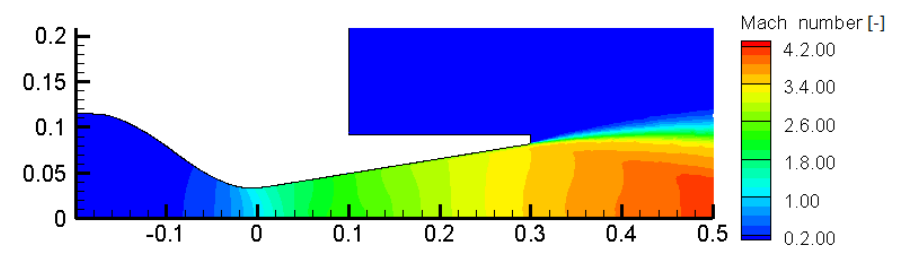

Fig. 5 Iso-Mach lines at $\mathrm{d}_{\mathrm{e}}=0.164 \mathrm{~m}$, conical nozzle

design points this time which are then used by the non-parametric regression to obtain the response surface. The pressure difference is plotted in Fig. 6. As with the previous case, the driving parameter in the optimal solution is the expansion criteria. The nozzle flow field is similar to the one produced by TOC nozzles, the internal shock system is clearly visible in Fig. 7. The smaller gradient in the contour at the exit means that the flow can better be oriented axially. The increase in thrust is around $1 \%$ compared to the conical nozzle from $12560.86 \mathrm{~N}$ to $12665.43 \mathrm{~N}$. The small diameters in both cases are due to the relatively small NPR value.

\section{Discussion}

The choice of a low nozzle pressure ratio in the optimization process was made due to the fact that the numerical mesh was only validated for this NPR region. It remains to be investigated how it performs in higher NPR ranges, which is necessary for practical applications. The main issue here is the flow field initialisation for extreme pressure differences, which might require an advanced initialisation technique.

\section{Conclusions}

In the present work the performance and feasibility of numerically optimized converging-diverging nozzle flows were investigated. The results from the numerical model compare well with the experimental and computational data and predict the correct flow structures and parameters with high confidence, reproducing the experimental data with $96.4 \%$ average accuracy. The optimization loop built upon the model was then tested on the diffusor of a one-parameter conical nozzle and a

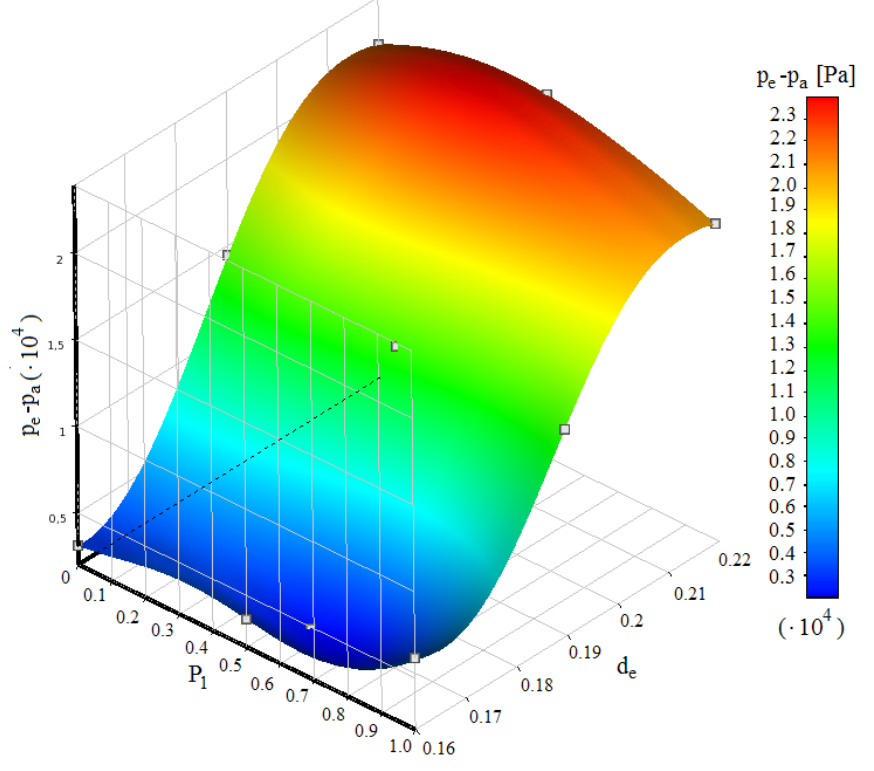

Fig. 6 Pressure difference response surface, bell nozzle

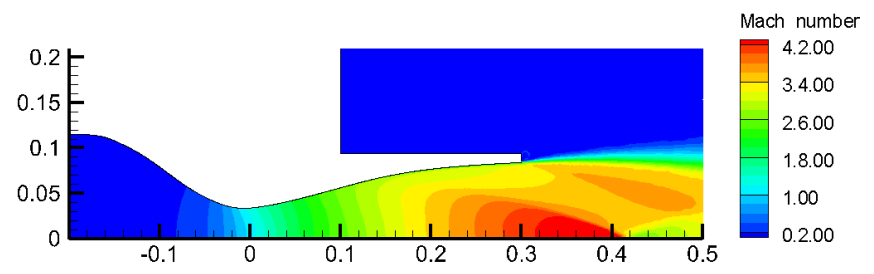

Fig. 7 Iso-Mach lines at $\mathrm{d}_{\mathrm{e}}=0.16825 \mathrm{~m} \mathrm{P}_{1}=0.5669$, bell nozzle

two-parameter bell nozzle to yield the largest obtainable thrust for the prescribed conditions. In both cases the method finds the optimum parameters and the resulting Mach contours and internal flow field structures are in good agreement with existing nozzle types. The method presented above is potentially extendable to include additional parameters and increase the polynomial order of the contour at the cost of increased computational cost. A natural extension is also the incorporation of gas species and combustion simulation instead of the homogenous air-operated model presented. A more advantageous addition would be a coupled stress and thermal analysis of the nozzle where the simulation data can be used to define a multiple-objective optimization in which the thrust is maximized while keeping the stress levels, nozzle weight and heat loads within certain limits.

\section{References}

Beneda, K. (2012). Numerical Simulation of MEMS-based Blade Load Distribution Control in Centrifugal Compressor Surge Suppression. In: $I C N$ PAA 2012 Congress: Mathematical Problems in Engineering, Aerospace and Sciences. American Institute of Physics, pp. 116-123.

Cai, G., Fang, J., Xu, X., Liu, M. (2007). Performance prediction and optimization for liquid rocket engine nozzle. Aerospace Science and Technology. 11(2), pp. 155-162.

https://doi.org/10.1016/j.ast.2006.07.002 
Guderley, G., Hantsch, E. (1955). Beste Formen für achsensymmetrische Überschallschubdüsen. (The Best Form for Axisymmetric Supersonic Nozzle.), Zeitschrift für Flugwissenschaften. 3(8). pp. 305-315. (in German)

Luedeke, H., Filimon, A. (2006). Axisymmetric Investigation of the VAC S6 Short Nozzle with Forced External Fluctuation ATAC-TEST CASE 1B. In: Proceedings of the ATAC-FSCD workshop, Noordwijk, The Netherlands.

Rao, G.V. R. (1958). Exhaust nozzle contour for optimum thrust. Journal of Jet Propulsion. 28(6), pp. 377-382. https://doi.org/10.2514/8.7324

Rao, G. V. R. (1960). Approximation of optimum thrust nozzle contour. ARS Journal. 30(6), p. 561

Rohács, D., Rohács J. (2016). Magnetic levitation assisted aircraft take-off and landing: (feasibility study - GABRIEL concept). Progress in Aerospace Science. 85, pp. 33-50.

https://doi.org/10.1016/j.paerosci.2016.06.001
Schweighofer, J., van der Meij, K., Gronarz, A., Hargitai, Cs.. Simongáti, Gy., (2015). Demonstration by simulation: The four simulator demonstrators of the FP7 EU project MoVeIT!. In: Proceedings of Hydrodynamics and simulation applied to Inland Waterways and Port Approaches Conference, Paris: Societe Hydrotechnique de France, Paper Schweighofer et al. ISBN:979-10-93567-08-2, $10 \mathrm{p}$.

Torngren, L. (2002). Correlation between Outer Flow and Internal Nozzle Pressure Fluctuations. In: Fourth Symposium on Aerothermodynamics for Space Vehicles. Vol. 487, p. 415. 